\title{
GeoAds: A Middleware Architecture for Music Service with Location-Aware Advertisement
}

\author{
Sheng-Po Kuo ${ }^{1}$, Shih-Ching Lin $^{1}$, Bing-Jhen $\mathrm{Wu}^{1}$, Yu-Chee Tseng ${ }^{1 *}$, and Chung-Chou Shen ${ }^{2}$ \\ ${ }^{1}$ Department of Computer Science \\ National Chiao Tung University, Taiwan \\ ${ }^{2}$ Computer \& Communication Research Labs \\ Industrial Tech. Research Inst., Taiwan
}

\begin{abstract}
This paper presents a middleware architecture to support location-based services (LBSs) based on heterogeneous localization systems. A GEOINFO gateway is implemented to hide the discrepancy of different localization systems from application developers. Hence, application developers can deploy their LBSs easily without considering the underlying localization techniques. To demonstrate the detailed process and advantages of the proposed architecture, we present a location-based service, called GeoAds, which is a streaming music service with geographical advertisement. This novel integration is expected to attract more mobile users' attention.
\end{abstract}

\section{Introduction}

Location-based services (LBSs) have emerged as one of the killer applications for mobile computing and wireless data services. While providing great market values to business applications, such services are also critical to public safety, transportation, emergency response, and disaster management. Only location without other context is meaningless. LBSs should be integrated with various contents or traditional services, such as multimedia and instant message service. Hence, this work considers a middleware architecture to bind traditional content and geographical information.

In this paper, we propose a middleware architecture to support LBSs in a large-scale environment. In such an environment, because every localization system has its limitation, the existence of heterogeneous localization systems is necessary. However, the complexity

${ }^{*}$ Y. C. Tseng's research is co-sponsored by Taiwan MoE ATU Program, by NSC grants 93-2752-E-007-001-PAE, 96-2623-7-009-002ET, 95-2221-E-009-058-MY3, 95-2221-E-009-060-MY3, 95-2219E-009-007, 95-2218-E-009-209, and 94-2219-E-007-009, by Realtek Semiconductor Corp., by MOEA under grant number 94-EC-17-A04-S1-044, by ITRI, Taiwan, by Microsoft Corp., and by Intel Corp. caused by the discrepancy of different localization systems should be transparent to application developers. Hence, a GEOINFO gateway is designed to simplify the inquiry process of geographical information. Finally, we will use a GeoAds service, which is a streaming music service with geographical advertisement, to demonstrate the operations of the proposed architecture.

\section{System Architecture}

A middleware architecture which consists of five system components is shown in Fig. 1. We consider an environment with heterogeneous communication infrastructure and localization systems. Each user equips with a mobile device which can be positioned. Our goal is to simplify the inquiry process of geographical information such that the deployment of LBSs can be effortless. For this purpose, the GEOINFO gateway is designed with two main features. First, it can accept remote procedure calls, named Terminal Location API, to support web services. Second, it can exchange well formulated XML messages with different location servers to acquire users' current locations. The function of each component will be explained in detail as follows.

\subsection{Location Servers}

To provide LBSs, many positioning technologies have been proposed. The Global Position System (GPS) is the most successful system so far, which is based on satellites to transmit signals to GPS receivers. However, in indoor environments, GPS cannot work properly because of buildings' sheltering effect. Hence, for indoor environments, many pattern-matching localization systems [3] are proposed. Such systems utilize existing wireless infrastructure to support localization. First, the received signal strength patterns of a set of training locations are collected. When an object is to be positioned, its received pattern is compared against those of the training locations to determine the object's location. 


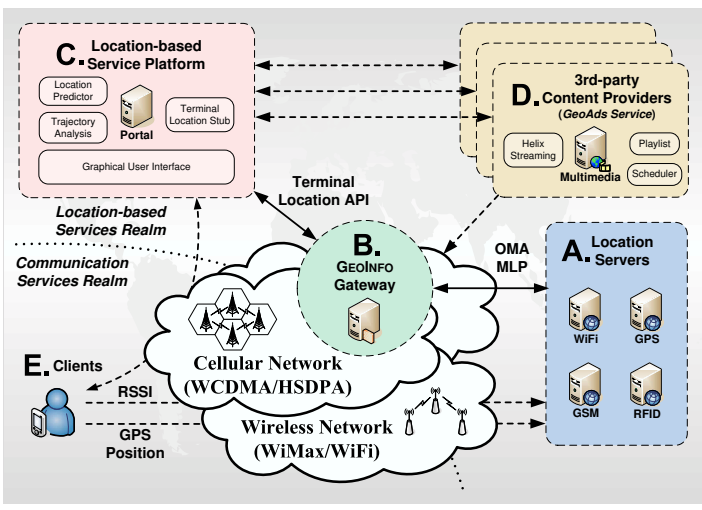

Figure 1. The proposed middleware architecture to support LBSs.

For a larger sensing field with both indoor and outdoor environments (such as a wireless city), mixed types of localization systems are preferred. Hence, we integrate GPS and pattern-matching localization techniques. In an outdoor environment without coverage of wireless infrastructure, the geographical information is provided by the GPS location server. Otherwise, we will rely on wireless infrastructures (such as $\mathrm{WiFi}$ or WiMAX) and pattern-matching solutions.

\subsection{GeoInfo Gateway}

In order to facilitate the location-related inquiry process for various applications, we propose a GEOINFo Gateway based on two standard specifications. One is the Mobile Location Protocol (MLP) published by Open Mobile Alliance (OMA) [4]. The other is Terminal Location API [6] which is one of Parlay X Web Services published by the Parlay Group [5].

Fig. 2 shows an example of the request and response messages of the basic Standard Location Immediate Service (SLIS). The Standard Location Immediate Request (SLIR) message is used to inquire the location of one or more mobile station identifiers (msid) within a set time. This message consists of a header part and a body part. In the header part, the requestor needs to explain its identity and password; in the body part, a list of msids is specified. An example is shown in Fig. 2(a), in which three types of msids are used. The queried result will be returned by the Standard Location Immediate Answer (SLIA) message. The geographical information can be described by many shapes defined in MLP. For example, as shown in Fig. 2(b), the first msid 001 is described by a circular shape, the second msid 002 is represented by a point, and the geographical information of the final msid 003 is not available so an error

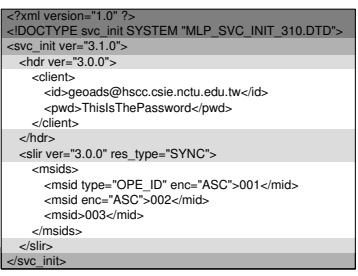

(a)

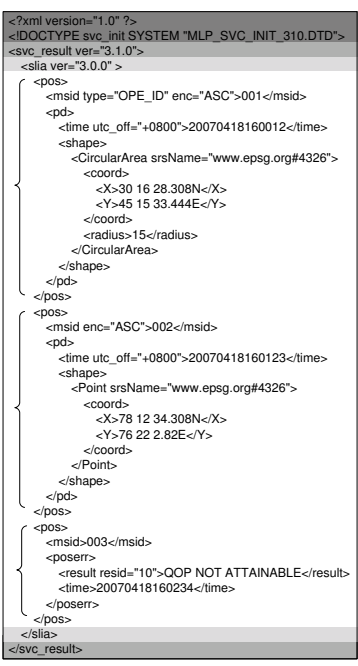

(b)
Figure 2. An example of OMA MLP messages: (a) The standard location immediate request (SLIR) message and (b) the standard location immediate answer (SLIA) message.

code is returned.

Although OMA MLP is a powerful message exchanging protocol, it is tedious for an application developer to compose requests and to interpret replied responses. Hence, a simple and general application programming interface is desired. The Parlay Group defines a series of powerful but simple web services for telecommunication industry. Among these specifications, Terminal Location can support inquiry of geographical information in terms of longitude, latitude, and altitude. For example, application developers can invoke the operation getLocation() and then receive the LocationInfo data structure, which contains the queried user's coordinate and the accuracy of this location information. The gateway takes the advantages of web services and provides application developers with users' locations easily no matter which programming language they use.

\subsection{Location-Based Service Platform}

The location-based service platform is a portal which accepts user requests for LBSs. Its main function is to provide graphical user interface and render the information obtained from 3rd-party content providers to users according to their current locations. The geographical information is retrieved through Terminal Location Stub 


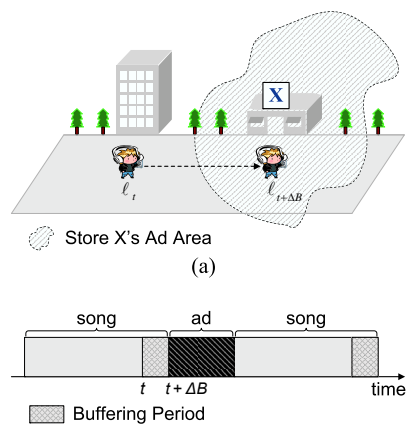

(b)

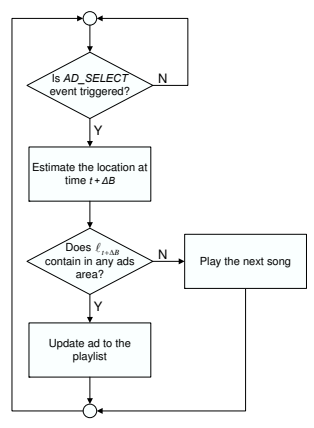

(c)
Figure 3. The scheduler of the GeoAds service: (a) scenario, (b) timeline, and (c) flowchart.

to connect the GEOINFO gateway. The queried results required by the Location Predictor and the Trajectory Analysis will be stored in the platform. The Location Predictor can predict the possible location of a user in the future and the Trajectory Analysis can provide the the analyses of the history of a user's traces.

\subsection{3rd-party Content Providers}

This component provides traditional content or services which are unaware of geographical information. For instance, to push guiding information to a user, the guiding service provider can ask the service platform to provide the next location of a user by the Location Predictor.

\subsection{Clients}

Users are expected to equip with mobile devices. The mobile devices are able to receive signal strengths from access points (APs) and have GPS receivers. Upon the Windows XP platform, we adopt the Wireless Research Application Programming Interface (WRAPI) [1] to collect signal strengths from nearby APs. The observed samples are transferred to the WLSN location server through 802.11 wireless networks. In an open space where satellites' signals can be received, the coordinates will be periodically reported to the GPS location server.

\section{Demonstration}

To demonstrate how the proposed middleware architecture works, we present the GeoAds service. GeoAds combines a streaming music service with the concept of geographical advertisement. Users can order their favorite songs through mobile devices. Between two songs, the system will push some location-related advertisements to the users.

Streaming is a technique that lets users continuously receive multimedia data. It needs buffering latency $\Delta B$. Hence, we need to decide the next clip (song or advertisement) before the end of the current one. Initially, users need to login their accounts and select their preferred song lists. There is a scheduler in the 3-rd party content provider which is responsible for notifying the streaming server the next suitable clip to be played. Fig. 3 a) shows a scenario where a user is at location $\ell_{t}$ at time $t$ and moves to $\ell_{t+\Delta B}$ at time $t+\Delta B$. The scheduler's timeline of this scenario is presented in Fig. 3 b). For each song, an AD_SELECT event is triggered by $\triangle B$ amount of time before the song ends. In this scenario, when the AD_SELECT event of the scheduler is triggered at time $t$, it will ask the service platform to get the user's location. Then, the service platform will retrieve the location by calling the procedure getLocation(). Furthermore, the scheduler will ask the service platform to predict that the user may move to location $\ell_{t+\Delta B}$ at $t+\Delta B$ and check if there is any suitable advertisement for that location. In this example, the 3rd-party streaming server will play store X's advertisement after the current song. In our implementation, RealNetworks' Helix server is adopted as the streaming server [2]. We can use the command

slta.bat 127.0.0.1 80 name password live.rm playlist

to play the clips indicated in the playlist text file. After receiving the notification from the service platform, the streaming server can dynamically adjust the streaming content by modifying the playlist file. Fig. 3(c) is the flowchart of the GeoAds service.

\section{References}

[1] A. Balachandran. Wireless Research API (WRAPI) website. http://sysnet.ucsd.edu/pawn/wrapi/

[2] Helix Server, RealNetworks. http://www. realnetworks.com/products/media_ delivery.html

[3] S.-P. Kuo, B.-J. Wu, W.-C. Peng, and Y.-C. Tseng. Cluster-Enhanced Techniques for Pattern-Matching Localization Systems. In IEEE MASS, 2007.

[4] Mobile Location Protocol v3.1. http: //www.openmobilealliance.org/release_ program/docs/MLP/OMA-LIF-MLP-V3_ $1-20040316-\mathrm{C} \cdot \mathrm{pdf}$

[5] The Parlay Group. http://www.parlay.org/en/ index.asp.

[6] Terminal Location API. http://www.parlay. org/imwp/idms/popups/pop_download. asp?Content ID $=11418$. 